\title{
The Large Earth-Based Solar Telescope
}

\author{
Oddbjørn Engvold \\ Institute for Theoretical Astrophysics \\ University of Oslo, Norway
}

Realising LEST, a truly next-generation solar telescope in terms of dimensions, innovative optics, and dome design, will mark a new era of international cooperation in solar physics and astrophysics.

The intricate fine-scale structure of the sun's magnetic field and the ceaseless variability of the solar atmosphere have been revealed these past decades using highquality solar telescopes such as the US National Solar Observatory's (NSO) $1.5 \mathrm{~m}$ Big Bear Solar Telescope on Kitt Peak, the $0.76 \mathrm{~m}$ NSO vacuum tower telescope on Sacramento Peak, the German refractor in Capri, the French solar refractor at Pic-duMidi, and more recently, the $0.7 \mathrm{~m}$ German and $0.5 \mathrm{~m}$ Swedish solar telescopes in the Canary Islands. Observational and theoretical discoveries strongly suggest that many observed solar phenomena (e.g., convection, turbulence, plasma flows, plasma waves, radiation, and magnetic instabilities) are tied to small-scale processes (Fig. 1) involving the magnetic fields within the sun's atmosphere. In general, our understanding of solar physics is largely founded on precise measurements attainable with high angular resolution and short integration times.

Ideas for a next-generation, high-resolution solar observing facility were conceived as a result of the growing demand for yet sharper and more precise imaging of the sun. One realised, even before any serious design concepts for a telescope had been put forward, that no single nation would possess sufficient financial and technical resources to design and build it. In 1969, an association of European solar physicists called the Joint Organization for Solar Observations (JOSO) was created with the goal of building a multinational, large-aperture solar telescope called LEST (Large European Solar Telescope). The members of JOSO immediately started an ambitious site survey programme, and about 10 years later grew to include non-European members and changed the name to the Large Earth-based Solar Telescope [1].

\section{Objectives}

The solar atmosphere constitutes a giant, natural plasma physics laboratory, where a host of known and new plasma physical processes can be studied providing instru-

Oddjbørn Engvold has been Professor of Astrophysics at the University of Oslo since 1989. Apart from a year at both Kitt Peak, USA, as a Visiting Astronomer and Sacramento Peak, USA, as a Fellow, he has been with the university since being a student. Professor Engvold, who was appointed in 1983 as the LEST Project Director, currently chairs the National Committee of the Norwegian Academy of Sciences.

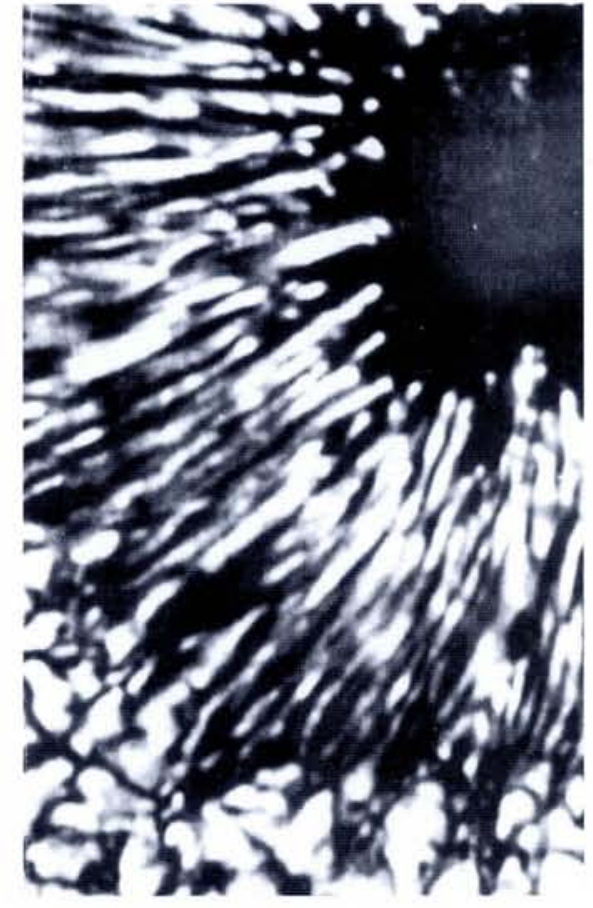

Fig. 1 - Whitelight image of a large sunspot - a solar magnetic storm - where luminous material is flowing at about $1 \mathrm{~km} / \mathrm{s}$ into the dark centre of the spot. The image, which has been filtered to enhance contrast, shows unprecedented fine structure along the light penumbral filaments, the narrowest discernible being a few hundred kilometres wide. [Courtesy of the Swedish Astrophysical Research Station, La Palma].

ments are built which have low instrumental polarization and an exceptional spatial resolution $(0.05-0.10$ arcsec or $35-70 \mathrm{~km}$ on the sun's surface) some five-times higher than at present. The $<0.10$ arcsec resolution is a threshold value since theory predicts that, below this limit, there are many observational possibilities for discriminating between theories to explain the fine structure of phenomena such as flares, sunspots and coronae. The probing of fine structure calls for a telescope with a large diameter, to provide enough photons for the required fine spectral analysis, and a low instrumental polarization so as not to distort the solar magnetic polarizations of spectral lines.

The last few years have seen a dramatic increase in observations on other stars of solar-type phenomena such as stellar flares, starspots, stellar activity cycles, stellar winds, chromospheres, and coronae.
Theoretical modelling of the stellar counterparts calls for a good understanding of the sun, which in turn depends on observations of the solar plasma fine structures with ultra-high spatial resolution. Indeed, the sun is perceived as the Rosetta Stone of astrophysics since it is the only star that can be studied at scales approaching those of many of its underlying processes.

Major scientific questions that LEST will address include:

- What is the nature of the transition from convective to radiative transport in a stellar atmosphere? Only the sun can show us how the transition between modes of transport varies from place to place over the surface of a star.

- How does the presence of a magnetic field modify convection, and how do fluid motions alter the structure of the magnetic field? Of particular interest are the differences between normal granular convection and granulation within magnetic regions.

- How is the fine-scale magnetic structure of the solar atmosphere related to the creation of the outer layers and to violent activity such as solar flares? Indeed, why is the atmosphere so finely structured at all? LEST will allow a close interaction between the theory of magnetic instability and the high resolution, high-precision polarimetric observations needed to test these theories.

\section{Project}

The LEST Foundation, is a non-profit organization created in 1983 to serve a major international collaboration in solar physics and astronomy by creating a new solar telescope that would surpass all existing instruments. It is domiciled, under Swedish law, in The Royal Swedish Academy of Sciences in Stockholm, and now comprises member organizations from Israel, Italy, Germany, Norway, Spain, Sweden, Switzerland, Australia, and the USA.

\section{Site}

In January 1990, the LEST Foundation Council selected a site within the Roque de los Muchachos observatory area on $\mathrm{La}$ Palma, Canary Islands. The site is located near the north-west rim of the Caldera, at altitude of $2350 \mathrm{~m}$ in the neighbourhood of the Swedish $0.5 \mathrm{~m}$ optical solar telescope and the British $4.2 \mathrm{~m}$ William Herschel stellar telescope (see cover illustration). The high optical quality is well documented from extensive experience with the Swedish vacuum tower telescope which is practically adjacent to the LEST site. The image qual- 
ity achieved with this telescope is widely recognised to exceed that of any other solar telescope as it frequently provides diffraction-limited performance for the $0.50 \mathrm{~m}$ aperture [2]. The site is unique and meets the scientific requirements for a 2.4 m nextgeneration telescope such as LEST.

\section{Requirements}

LEST is conceived as a comprehensive, high resolution, low-polarization facility for observations of the sun in the visible and near-infrared wavelengths. The major design requirements are:

- High angular resolution $(<0.1$ arcsec) implying a large aperture ( $>2 \mathrm{~m}$ diameter) giving near diffraction-limited performance.

- Ability to perform polarization measurements with an accuracy $\Delta p / p \approx 10^{-3}$.

- High photon flux in order to achieve short integration times of small-scale features.

- Ability to maintain uniform resolution for periods of time comparable to, or greater than, the typical development time of smallscale solar phenomena.

- Broad spectral coverage - from the absorption edge of the atmosphere (wavelength $\lambda \approx 300 \mathrm{~nm})$ to the near-infrared $(\lambda \leq$ $2.5 \mu \mathrm{m})$.

Being a ground-based facility, LEST will provide considerable flexibility for postfocus instrumentation by allowing for experimental set-ups as well as major, essentially permanent, instruments. One must furthermore assume that LEST will cater for a broad international users' group. Yet by being an autonomous installation LEST will also provide remote access via computer terminals in the users' home institutions.

\section{Design}

The LEST project reached a milestone in 1990 with the review and approval by the LEST Foundation of the Phase B Design Study. The design is revolutionary in many ways and can be regarded as representing a next-generation device, both in terms of dimensions, innovative optics, and dome design. The telescope is a modified Gregorian type with an additional concave mirror behind the primary mirror (Fig. 2 shows the optical layout). More than $99 \%$ of the energy is absorbed by a heat rejector in the primary focus of the parabolic $f / 2.3$ main mirror. The third mirror forms an f/75 beam, and reimages the Gregorian focus at the large instrument table in base of the telescope (Fig. 3). The same mirror also forms an image of the pupil at the position of the flat mirror M5 which will be the system's fast guider, agile mirror.

Most modern solar telescopes have an evacuated light path to eliminate internal seeing. However, the thermal and mechanical stresses in the entrance and exit windows are a major concern and all solutions have drawbacks [3]. Using windows will also limit the telescope aperture to less than $1 \mathrm{~m}$ and the longest wavelength that can be measured to $\leq 2.5 \mu \mathrm{m}$ owing to absorption in the entrance window.

The idea of employing helium cooling has been explored through several practical studies [4], and more recently by measurements with a full-scale model of the tube which will house the optical components.

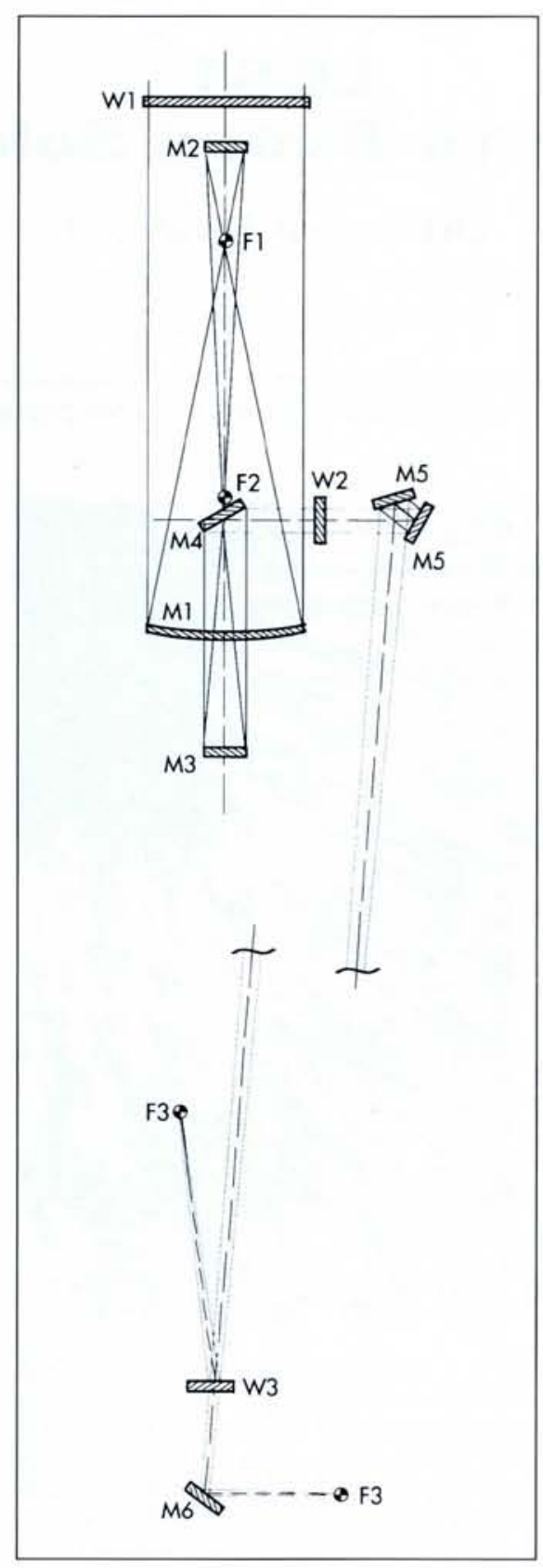

They have demonstrated that a circulating helium atmosphere allowing a thin, highquality optical window to be used eliminates the serious problem of telescope seeing.

\section{Stokes polarimeter}

As the measurement of magnetic fields is a principle scientific objective, the photon flux requirement for Stokes polarimetry has been a fundamental design consideration. The polarization modulator of the polarimetry system will be inserted into the light beam prior to the first inclined reflection. Its location is immediately before the secondary image plane of the modified Gregorian telescope. A high frequency $(50-100 \mathrm{kHz})$ piezo-elastic modulator (PEM) will be used in conjunction with a demodulation scheme based on synchronous shifting of charges in a modified charge coupled device (CCD) in the instrument focus [5]. The problem of combining the large-scale CCD array detector having slow readout with the much faster electroptic polarization modulators
Fig. 2 - Layout of LEST's optical systems. The main telescope is of the Gregorian type with an additional concave mirror M3 behind the deformable main mirror M1. Light passes through the entrance window $W 1$, leaves the telescope tube through W2, and is diverted by the fast, agile flat mirror M5 to mirror M6 on the instrument platform where a focus F3 is formed at instruments. Light reflected off the exit vacuum window W3 is fed to a wavefront sensor which is used in active and adaptive optics system to adjust focus (M3), alignment and aberration (M1), and seeing (M5).

has been solved by having several image planes within a single CCD chip.

\section{Structure}

A large, $19.5 \mathrm{~m}$ in diameter, rotating platform at the bottom of the telescope tower provides adequate space for at least 15 focal plane instruments (Fig. 3); large, vertically mounted equipment, notably a high dispersion spectrograph, will be housed in a $4 \mathrm{~m}$ diameter, $12 \mathrm{~m}$ deep, vertical steel cylinder beneath the platform. Full compensation for the field rotation is achieved by regulating the relative motion of the platform and the mirror M6.

The compact telescope and the integrated mounting is placed on top of the single, conically shaped, concrete tower which is about $25 \mathrm{~m}$ tall to minimise the influence of air turbulence near the ground. The compact, lightweight, spherical dome and the telescope tube protect the telescope from radiative heating and weather, and the overall aerodynamic shape minimises wind buffeting.

\section{High-Resolution Imaging}

Active optical systems control a telescope's subsystems to correct errors inherent in the telescope structure. The successful implementation of the active figure-controlled main mirror of the European Southern Observatory's New Technology Telescope (NTT) suggests that a similar meniscus-type, deformable main mirror should be developed for LEST. Consequently, LEST's main mirror will be supported in the axial direction by 81 individually regulated points and by three actuators to control tilt, and in the transverse direction by 24 regulated points and another three actuators.

The tight positional and alignment tolerances of the primary and secondary mirrors call for a continuously monitored correcting system. A low spatial and temporal resolution Hartmann-Shack type wavefront sensor with correlation trackers will be adopted, where the sensor uses light reflected off the exit vacuum window near focus. It is foreseen that this system will be used to sense and update the mirror alignment and to check the figure of the main mirror once every several minutes, without interfering with observations.

Owing to the good seeing quality of the La Palma site, LEST is expected to give near-diffraction limited imaging in the nearinfrared even without an adaptive optics (AO) system similar to those now becoming available in astronomy to correct for image degradation caused by external factors. 


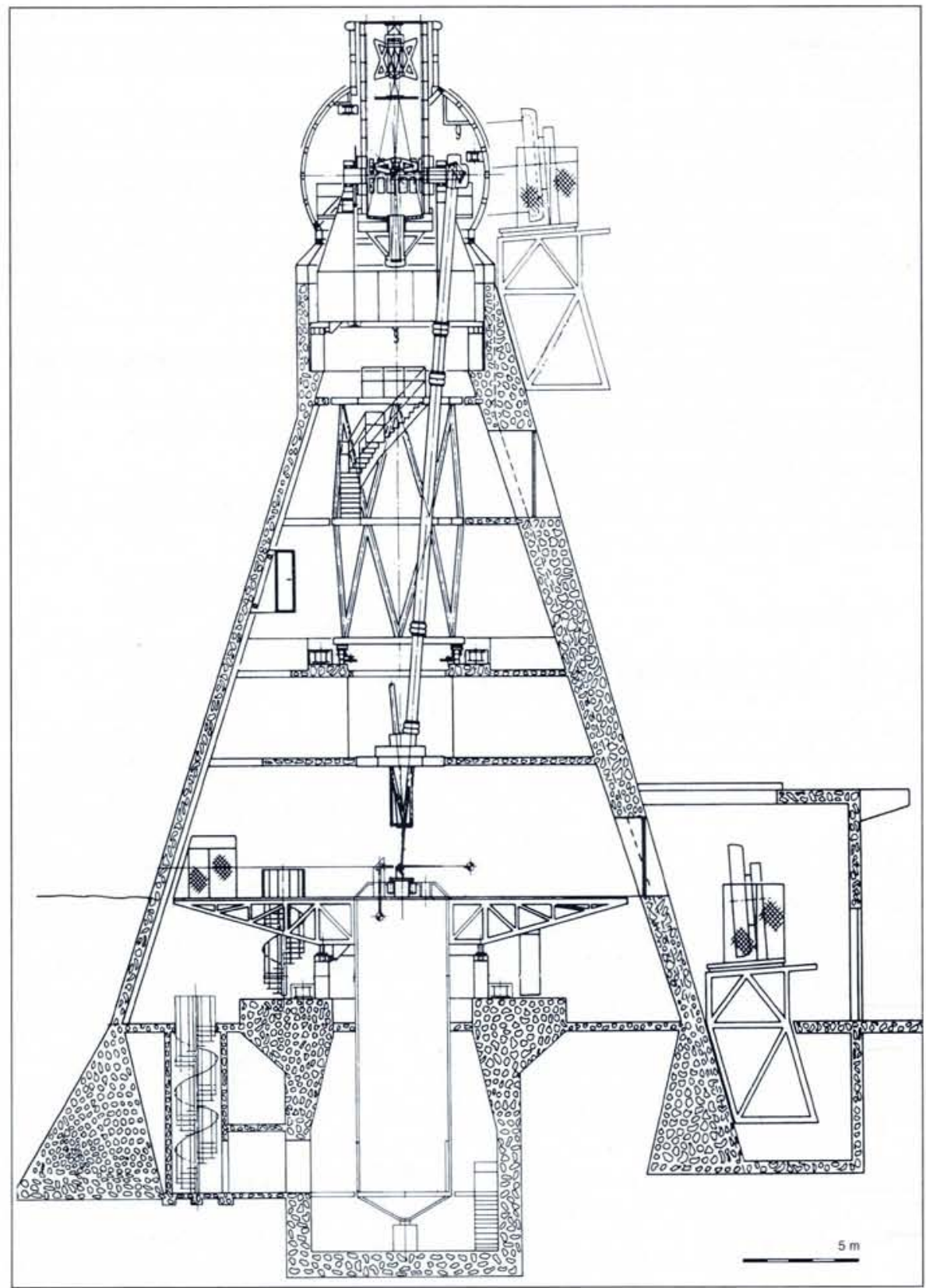

Fig. 3-Cross-section of the LEST telescope and tower structure. The telescope tube at the top of the tower is shown pointing vertically upwards. Note the nearly vertical tube taking the light beam to the instrument platform at ground level, with the vertical instrument cylinder mounted underneath.

\section{LEST \\ Main Design Features (from [7])}

Large aperture:

Main mirror:

Field of view:

Spectral range:

Spatial resolution:

Light path:

Outer telescope shell:

Operation and data handling:

Stokes polarimetry:

Correction of seeing:

Control of telescope aberrations:

Stabilisation of image motion produced by telescope vibration and seeing:

Permanent and experimental post-focus instruments (non-rotating field-of-view): mounted on optical benches on a large rotating table
However, there is little doubt that such a system will be used, the question being instead when it will be available, whereupon LEST will be unsurpassed for high-resolution observations in the near infrared. Recent tests made with the NSO vacuum tower telescope at Sacramento Peak, USA, using an $A O$ system built by a group from Lockheed Corp., USA, gave promising results. LEST's system will be an integral part of the design [6], with the deformable mirror and the fast tilt-mirror located at the image of the pupil on the elevation axis outside the telescope tube immediately above mirror M5.

\section{Status}

The Project Technical Group responsible for the design study is primed to embark upon the construction phase. With the requisite funding by various national agencies, this phase would begin at the end of 1994 with completion in 1998. The advanced stage of development has meant that LEST ranks highly among projects recommended for national funding. Thus, the report of the Joint Working Group on Groundbased Astronomy by representatives of national research councils in Canada, France, Germany, Italy, Japan, the UK, and the USA said that "the LEST project is one of major importance for the solar physics community and one which can proceed only by international collaboration". The Bahcall report Astronomy in the USA for the 1990's and the European Physical Society report Solar Physics in Europe recommended a strengthening of solar ground-based research facilities.

\section{Conclusions}

The Large Earth-based Solar Telescope will provide the world's solar physics community with a powerful, next-generation solar telescope with unprecedented angular resolution and highly accurate polarimetry. Essentially unique, LEST is designed to tackle from the ground the difficult and pressing observational tasks in solar research during the 1990's and well into the next century.

The optical design is a $2.4 \mathrm{~m}$ aperture, polarization-free concept based on a modified Gregorian system. An actively controlled NTT-type main mirror, a high-precision pointing and tracking system, a heliumfilled light path with thin windows, and an integrated adaptive optics system, will provide near diffraction-limited performance.

[1] Godoli G., LEST Annual Report (1990) p. 7; Stenflo J.O., Vistas in Astronomy 28 (1985) 571.

[2] Scharmer G.B., Solar \& Stellar Granulation, Eds.: R.J. Rutten and G. Severino (Kluwer) 1989, p.161; Wyller A.A. and Scharmer G.B., Vistas in Astronomy 28 (1985) 467.

[3] Dunn R.B., LEST Tech. Report No. 3 (1984); Solar Physics 100 (1985) 1.

[4] Engvold O., et al., Appl. Optics 22 (1983) 10.

[5] Povel H., Aebersold H., and Stenflo J.O., LEST Tech. Report No. 40 (1989).

[6] Dunn R.B., LEST Tech. Report No. 28 (1987) p. 243.

[7] Engvold O. and Andersen T., LEST Design, Report of the LEST Foundation (1990). 